\title{
Preparation of Nickel-Copper Bilayers Coated on Single-Walled Carbon Nanotubes
}

\author{
Zhong Zheng, ${ }^{1}$ Shan Zhao, ${ }^{1}$ Shijie Dong, ${ }^{1}$ Lianjie Li, ${ }^{1}$ Anchun Xiao, ${ }^{2}$ and Sinian Li ${ }^{1}$ \\ ${ }^{1}$ Hubei Provincial Key Laboratory of Green Materials for Light Industry, Hubei University of Technology, Wuhan 430068, China \\ ${ }^{2}$ School of Science, Hubei University of Technology, Wuhan 430068, China
}

Correspondence should be addressed to Zhong Zheng; zhengzh215@163.com and Shijie Dong; dongsjsj@163.com

Received 31 December 2014; Accepted 18 February 2015

Academic Editor: Stefano Bellucci

Copyright (c) 2015 Zhong Zheng et al. This is an open access article distributed under the Creative Commons Attribution License, which permits unrestricted use, distribution, and reproduction in any medium, provided the original work is properly cited.

Due to oxidizability of copper coating on carbon nanotubes, the interfacial bond strength between copper coating and its matrix is weak, which leads to the reduction of the macroscopic properties of copper matrix composite. The electroless coating technics was applied to prepare nickel-copper bilayers coated on single-walled carbon nanotubes. The coated single-walled carbon nanotubes were characterized through transmission electron microscope spectroscopy, field-emission electron microscope spectroscopy, Xray diffractometry, and thermogravimetric analysis. The results demonstrated that the nickel-copper bilayers coated on singlewalled carbon nanotubes possessed higher purity of unoxidized copper fine-grains than copper monolayers.

\section{Introduction}

Carbon nanotubes (CNTs) are composed of quasi circular tubes with unique polygonal carbon-ring microstructural units. Carbon atoms in CNTs skeleton are linked together by $\mathrm{sp}^{2}$ hybrid $\mathrm{C}=\mathrm{C}$ covalent bonds. And they have excellent electrical, thermal, and mechanical performance $[1,2]$, as well as high structural and chemical stability. They have potential value in many areas such as nanoelectricals, structural and functional composite materials [3-9]. CNTs can be classified into single-walled carbon nanotubes (SWCNTs) and multiwalled carbon nanotubes (MWCNTs) according to the numbers of their graphene layers. The coaxial layers of MWCNTs are among several layers to dozens of layers. They are easy to bridge among adjacent walls and full of small hole-defects. SWCNTs consist of a single cylindrical graphite sheet with fewer defects. The calculated thermal conductivity of MWCNTs is about $3000 \mathrm{~W} / \mathrm{mK}$, while that of SWCNT is larger than $4000 \mathrm{~W} / \mathrm{mK}$ [10-12]. Therefore, SWCNTs are more appropriate for preparation of composites with high thermal conductivity and electrical conductivity through the use of CNTs as strengthening phase.

Both the wettability and bonding strength of interfaces between copper matrix and CNTs are weak. Both physicochemical properties of interface regions, such as elastic modulus, thermal conductivity, linear expansion coefficient, and thermodynamic parameters, are discontinuous. The discontinuousness leads to reduction of electrical conductivity, thermal conductivity, thermal expansion composites, stress distribution, strain distribution, and load transfer.

Some methods such as coarsening phase surfaces, addition of alloying elements, and interlayers deposition are utilized to improve interfacial bond strength $[13,14]$. The coarsening phase surfaces and addition of alloying elements could destroy SWCNTs or introduce impurities. The methods of depositing interlayers include electroless coating, sol-gel technology $[15,16]$, chemical vapor deposition (CVD) [17, $18]$, and physical vapor deposition (PVD) $[19,20]$. Sol-gel technology, CVD, and PVD are dependent on expensive equipment. An adhesion layer must be fabricated before using CVD or PVD to deposit copper because of the poor adhesion of copper. That process is complex. Electroless coating process features easy operation, high stability, and reliability with simple equipment. Homogeneousness and deepening of coating can be obtained without damage of substrate. Therefore, the electroless coating method is often applied to prepare copper layers on the surface of CNTs. For instance, Daoush et al. [3] and Khaleghi et al. [9] prepared copper coating on the surface of SWCNTs through electroless plating process successfully. 
However, due to high oxygen affinity of copper, the coating is prone to be oxidized and to exist in forms of copper oxides during the process of electroless coating, preparing composites, heating, and storage. Copper oxides coating on SWCNTs can be applied to the catalytic effect, such as photocatalytic degradation [21], water splitting [22], and gas sensing applications [23]. Nevertheless, they would decrease the performance of copper coating in electrical and thermal conductivity and the bonding strength of interfaces between SWCNTs and copper matrix.

Aiming at the problems mentioned above, some heat treatments have been adopted to reduce copper oxides into pure copper of coating under reducing atmosphere [24, 25]. Xue-xi Zhang et al. disclosed a preparation method in Chinese Invention Patent entitled "The Preparation Method of Carbon Nanotubes-Copper Oxide Composite Powder" [24]. They coated CNTs with copper oxides ( $\mathrm{CuO}$ and $\mathrm{Cu}_{2} \mathrm{O}$ ) through electroless plating process, then reduced sedimentary copper oxides into pure copper, and crystallized them simultaneously in hydrogen atmosphere at $200^{\circ} \mathrm{C}$. The obtained pure copper coating was continuous and uniform with appropriate thickness. However, the technique needs to flux flammable and explosive gas, such as hydrogen, which is difficult to be controlled.

At present, few related researches on oxidation of the electroless copper coating on SWCNTs were reported. Jinmei Wang [26] compared electroless copper coating with nickel coating and $\mathrm{Ni}-\mathrm{Cu}$ composite coating on the surface of CNTs. Wang found that the high-temperature resistance and oxidation resistance of $\mathrm{Ni}-\mathrm{Cu}$ composite coating lie between those of the copper coating and nickel coating. In addition, Kiani [27] deposited copper and nickel onto the carbon fiber surface through electroless coating and examined the mechanisms of high-temperature oxidation of copper and nickel-coated carbon fibers. Kiani found that copper coating reduced the temperature of the fiber oxidation, and nickel coating increased the minimum temperature needed for this oxidation reaction. Uncoated and copper-coated fiber oxidation gives rise to $\mathrm{CO}_{2}$. However, the oxidation of nickelcoated fibers produces $\mathrm{CO}$, because of the protective nature of the oxidized nickel coating, which limits oxygen access to the carbon fibers.

To improve the oxidizability of copper coating on SWCNTs, the three-step-refluxing purified SWCNTs were deposited with nickel through electroless coating at first and then deposited copper. The morphologies, structure, and composition distribution of electroless coating on SWCNTs were determined by some modern analytical techniques and characteristic methods. The Ni-Cu bilayers on SWCNTs possessed unoxidized copper with high electrical and thermal conductivity which could bond SWCNTs to copper matrix firmly. Therefore, the Ni-Cu bilayers on SWCNTs were suitable for the application in copper matrix composite to achieve desirable macroeconomic performance of composite.

\section{Experimental}

SWCNTs synthesized through chemical vapor deposition (CVD) were obtained from Chengdu Organic Chemicals Co.,
Ltd., Chinese Academy of Sciences. Analytical reagents, such as nitric acid, $\mathrm{NiSO}_{4} \cdot 6 \mathrm{H}_{2} \mathrm{O}, \mathrm{CuSO}_{4} \cdot 5 \mathrm{H}_{2} \mathrm{O}$, and $\mathrm{C}_{2} \mathrm{H}_{2} \mathrm{O}_{3}$, were commercially available. A CS-OY-CQ30 probe ultrasonic generator was employed to disperse SWCNTs before purification (Beijing Heng Odd Instrument Co., Ltd.). A VOSHIN96IIL bath ultrasonic generator was used to disperse SWCNTs during electroless plating (Wuxi Voshin Instrument Co., Ltd.). Samples were filtered by $0.45 \mu \mathrm{m}$ Millipore filters.

The samples were characterized through a field-emission scanning electron microscope (FE-SEM, Quanta 450) equipped with an energy dispersive spectroscopy analyzer (EDS, SUPRA 55 SAPPHIRE), a transmission electron microscope (TEM, HITACHIH-7000FA), and an X-ray diffractometer (XRD, XD-2). To evaluate the samples, thermogravimetric analysis was conducted in airflow with a heating rate of $10^{\circ} \mathrm{C} \cdot \mathrm{min}^{-1}$ using a thermogravimetric analyzer (TGA, SDT Q600). follows.

The detailed experimental procedures are presented as

2.1. Surfactant-Aided Ultrasonic Dispersion. SWCNTs have large aspect ratio and high polarizability. Electrostatic attraction and van der Waals forces among them lead to agglomeration and their easy tangling. The energy induced through ultrasonic vibrations at a high frequency enables matted SWCNTs to overcome these interactions. In particular probetype ultrasonicators (their ultrasonic tips can deliver more energy to SWCNTs) and their dispersion effect were better than those of ultrasonic baths at the same frequency [28]. In addition, the exploration of dispersion mechanism has shown that $\pi-\pi$ stacking interaction formed through surfactants adsorbed on the surface of SWCNTs is stronger than interaction among SWCNTs [29].

Therefore, probe-type ultrasonication has been employed for surfactant-aided dispersion of SWNTs into a solution in the study.

A $0.025 \mathrm{w} / \mathrm{v} \%$ of initial SWCNTs was dispersed in a $0.35 \mathrm{w} / \mathrm{v} \%$ SDBS aqueous solution through ultrasonication for $30 \mathrm{~min}$. The ultrasonic dispersion employs a probe ultrasonic generator with a frequency of $35 \mathrm{kHz}$ and a power of $60 \mathrm{~W}$, and it stands for $5 \mathrm{~s}$ after ultrasonic treatment for $5 \mathrm{~s}$ in each 10 s cycle.

2.2. Refluxing Purification. Commercial SWCNTs synthesized by CVD contain some nanometer impurities including Fe catalyst particles, amorphous carbon, and graphite flakes. These impurities could barrier coating and modify the excellent performance of SWCNTs. Thus they must be removed from the samples before electroless coating.

More mild conditions were chosen during purifying to prevent damage or loss of SWCNTs. The complete purification procedure had three steps. The first one was refluxing a $0.035 \mathrm{w} / \mathrm{v} \%$ SWCNTs solution in $6 \mathrm{M}$ nitric acid at $118^{\circ} \mathrm{C}$ for $5 \mathrm{~h}$, followed by deionized water washing; the second one was refluxing in $6 \mathrm{M}$ sodium hydroxide at $118^{\circ} \mathrm{C}$ for $3 \mathrm{~h}$, followed by deionized water washing; and the third one was refluxing in $6 \mathrm{M}$ hydrochloric acid at $118^{\circ} \mathrm{C}$ for $3 \mathrm{~h}$, followed by deionized water washing. Finally, the sample was dried under vacuum for $3 \mathrm{~h}$ at $80^{\circ} \mathrm{C}$. 


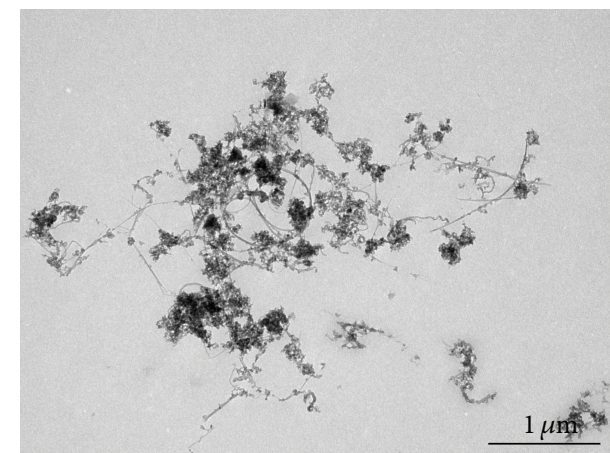

(a)

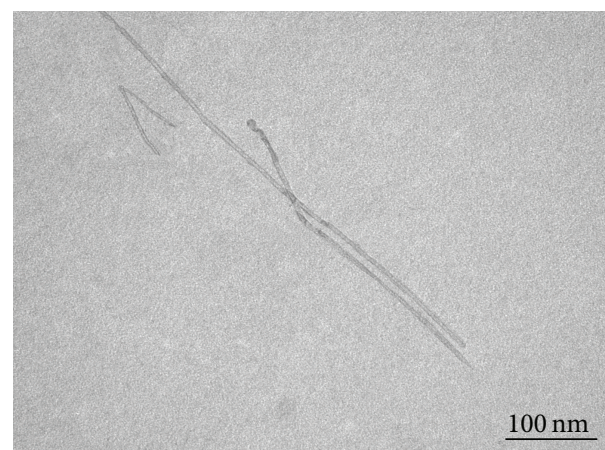

(b)

FIGURE 1: Representative TEM images of (a) initial and (b) purified SWCNTs.

TABLE 1: Composition of nickel electroless plating bath.

\begin{tabular}{lcccc}
\hline Chemical & $\mathrm{NiSO}_{4} \cdot 6 \mathrm{H}_{2} \mathrm{O}$ & $\mathrm{NaH}_{2} \mathrm{PO}_{2}$ & $\mathrm{Na}_{3} \mathrm{C}_{6} \mathrm{H}_{5} \mathrm{O}_{7}$ & $\mathrm{NH}_{4} \mathrm{Cl}$ \\
\hline $\begin{array}{l}\text { Concentration/ } \\
\left(\mathrm{mol} \cdot \mathrm{L}^{-1}\right)\end{array}$ & 0.13 & 0.18 & 0.19 & 0.56 \\
\hline
\end{tabular}

2.3. Sensitization and Activation. It is very difficult for metals or compounds to deposit on the surface of SWCNTs because of the inherent hydrophobicity and low chemical activity of SWCNTs [30]. So the surface of the SWCNTs must be activated to form catalytic nuclei at first. These nuclei then initiate the redox reaction to make metals deposit and grow up on it. And then continuous coating was obtained.

The detailed procedures are as follows. A $0.025 \mathrm{w} / \mathrm{v} \%$ of purified SWCNTs was activated in an aqueous solution of $0.1 \mathrm{M} \mathrm{SnCl}_{2} / 0.1 \mathrm{M} \mathrm{HCl}$ through ultrasonic dispersion for $10 \mathrm{~min}$, followed by deionized water washing. Then it was sensitized in an aqueous solution of $0.014 \mathrm{M} \mathrm{PdCl}_{2} / 0.25 \mathrm{M}$ $\mathrm{HCl}$ through ultrasonic dispersion for $8 \mathrm{~min}$, followed by deionized water washing.

2.4. Ni-Cu Electroless Plating. A $0.03 \mathrm{w} / \mathrm{v} \%$ of activated SWCNTs was dispersed in an electroless nickel plating bath (composition as shown in Table $1, \mathrm{pH}=8.5$ ) through ultrasonication at $25^{\circ} \mathrm{C}$ for $15 \mathrm{~min}$, followed by deionized water washing.

Then it was dispersed in an electroless copper plating bath (composition as shown in Table 2, $\mathrm{pH}=11.5$ ) through ultrasonication at $30^{\circ} \mathrm{C}$ for $25 \mathrm{~min}$, followed by deionized water washing. Finally, the sample was dried under vacuum for $3 \mathrm{~h}$ at room temperature.

2.5. Electroless Copper Coating. For comparison, SWCNTs were deposited with copper only through the aforementioned electroless coating process.

\section{Results and Discussion}

3.1. TEM Analysis. The samples for TEM were prepared through dropping the ethanol suspension of SWCNTs onto a grid which was left to air-dry. TEM images before and after purification (see Figure 1) show clearly the effectiveness of our three-step-refluxing purification processes. TEM image of initial sample (see Figure 1(a)) clearly shows entangled SWCNTs bundles in a mixture with metal nanoparticles (black dots), amorphous carbon encasing metal particles, and free graphite particles. The SWCNTs are typically $6 \mathrm{~nm}$ in diameter and 5 30 Mm in length. The size of metal particles ranges from ten to several ten $\mathrm{nm}$. Long SWCNTs with high structural quality are still observed in the TEM micrographs of purified samples (see Figure $1(\mathrm{~b})$ ). It reveals that the purification treatments can remove most of the metal particles and carbonaceous impurities without significant damage to SWNTs.

3.2. SEM and EDS Analyses. Some representative SEM micrographs of copper deposited SWCNTs, Ni deposited SWCNTs, and $\mathrm{Ni}-\mathrm{Cu}$ deposited SWCNTs are shown in Figures 2(a), 2(b), and 2(c), respectively. A comparison among them indicates significant differences in the morphology of the three samples. Ni-Cu deposited SWCNTs are 230 270 nm in diameter. The coating shows compact fine-grain appearance without obvious drusy structure. Copper deposited SWCNTs are 140 210 $\mathrm{nm}$ in diameter. The grains of copper monolayers grew in clusters and were inhomogeneous in size. There were quite a few vacancies among those coarse grains. The distribution of Ni-Cu deposition is more complete, homogenous, and continuous than those of copper deposition. Besides, $\mathrm{Ni}$ deposited SWCNTs are 120 150 nm in diameter. The distribution of thick Ni-coating is also complete, homogenous, and continuous.

These phenomena may be related to the poor interfacial bonding between SWCNTs and reactive sites (to catalyze $\mathrm{Pd} / \mathrm{Sn}$ colloidal particles) on them, which could cause reactive sites to migrate and coalesce. In addition, reactive sites on the surface of SWCNTs were discontinuous. When copper was deposited on the reactive sites, some coalescing of copper grains also took place. After nickel has deposited over the reactive sites on SWCNTs, it could fully grow along normal direction and tangent direction on the surface of SWCNTs due to its strong autocatalysis. Finally nonactivated areas on the surface of SWCNTs were covered with continuous and uniform coating by the way of tangential growth. Ni substrate, as a catalyst, was unable to migrate, so deposited copper 
TABLE 2: Composition of copper electroless plating bath.

\begin{tabular}{lccccc}
\hline Chemical & $\mathrm{CuSO}_{4} \cdot 5 \mathrm{H}_{2} \mathrm{O}$ & $\mathrm{C}_{2} \mathrm{H}_{2} \mathrm{O}_{3}$ & EDTANa $_{2}$ & $\mathrm{NaKC}_{4} \mathrm{H}_{4} \mathrm{O}_{6} \cdot 4 \mathrm{H}_{2} \mathrm{O}$ & $2,2^{\prime}-\mathrm{Bipyridine}$ \\
\hline Concentration $/\left(\mathrm{mol} \cdot \mathrm{L}^{-1}\right)$ & 0.03 & 0.2 & 0.24 & 0.05 & $10\left(\mathrm{mg} \cdot \mathrm{L}^{-1}\right)$ \\
\hline
\end{tabular}

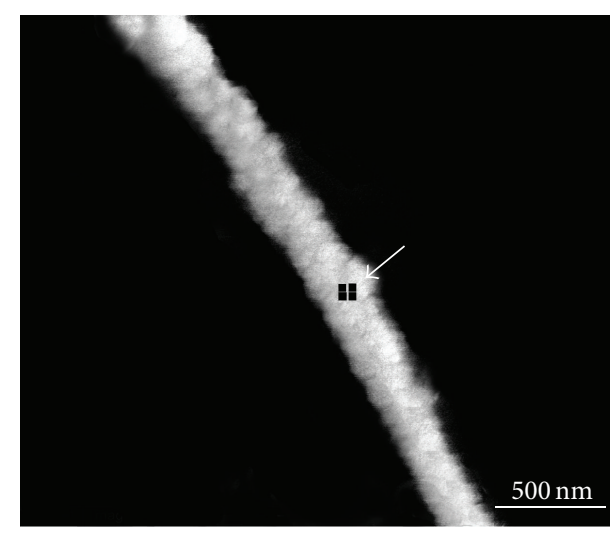

(a)

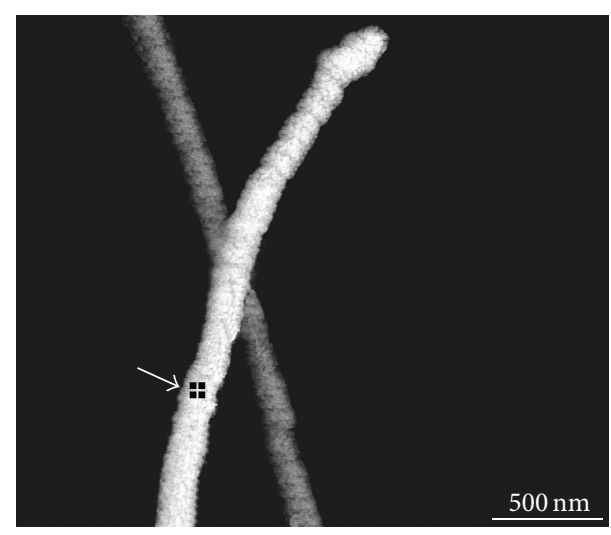

(b)

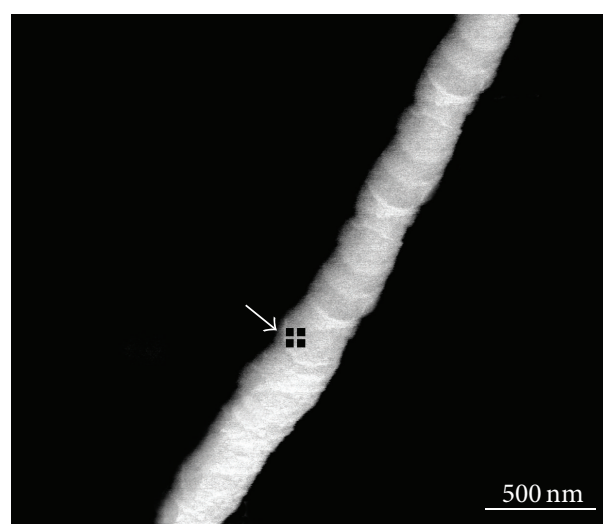

(c)

FIGURE 2: Representative SEM images of (a) Cu deposited SWCNTs, (b) Ni deposited SWCNTs, and (c) Ni-Cu deposited SWCNTs.

over it would not coalesce. On the other hand, the entire surface of Ni substrate has catalytic activity and was relatively smooth. Copper could nucleate homogeneously on it; thus high density nuclei and fine-grains of copper were obtained.

Figures 3(a), 3(b), and 3(c) show the EDX spectra for copper deposited SWCNTs, Ni deposited SWCNTs, and NiCu copper deposited SWCNTs corresponding to Figures 2(a), 2(b), and 2(c), respectively. All of the profiles show peaks for copper or nickel along with peaks for carbon and oxygen. They indicate that our electroless deposition processes were efficient at achieving nickel or copper deposit on the surface of SWNTs. Part of the observed oxygen might be the air trapped inside the SWCNTs or coating; the remaining part might be attributed to the oxidized coating. Table 3 shows the tabulated element content values for copper deposited SWCNTs, Ni deposited SWCNTs, and Ni-Cu deposited SWCNTs. The copper-to-carbon fraction of copper deposited SWCNTs sample is $14.86 \%$ in atom, while that of Ni-Cu deposited SWCNTs sample is $25.41 \%$ in atom, indicating the latter coating is thicker, as observed through SEM. And the oxygen-to-copper fraction of copper deposited SWCNTs is $59.23 \%$ in atom, while that of Ni-Cu deposited SWCNTs is $23.86 \%$ in atom, indicating that the former $\mathrm{Cu}$-coating is oxidized more seriously.

3.3. X-Ray Diffraction. The XRD patterns of purified, copper deposited SWCNTs, Ni deposited SWCNTs, and Ni-Cu deposited SWCNTs and over the range of angle shift values of $10^{\circ}$ through $90^{\circ}$ are shown in Figure 4 . In the diffraction pattern $(2 \theta)$ of purified sample corresponding to Figure 4(a), two prominent peaks are observed at approximately 26.2 and 43.2. They are due to the (llll $\left.\begin{array}{lll}0 & 0 & 2\end{array}\right)$ and $\left(\begin{array}{lll}1 & 0 & 0\end{array}\right)$ planes of CNTs, respectively.

In the diffraction pattern $(2 \theta)$ of copper deposited SWCNTs corresponding to Figure 4(b), five peaks are observed at approximately $32.6,41.2,56.3,62.21$, and 73.26 , respectively. The peaks at 41.2, 56.3, and 62.21 were assigned to the $\left(\begin{array}{lll}1 & 1 & 1\end{array}\right),\left(\begin{array}{lll}2 & 0 & 0\end{array}\right)$, and (2 220$)$ planes of the fcc phase of copper, respectively. The remaining peaks at 32.6 and 62.21 correspond to $\mathrm{CuO}$ and $\mathrm{Cu}_{2} \mathrm{O}$, respectively. The growth 


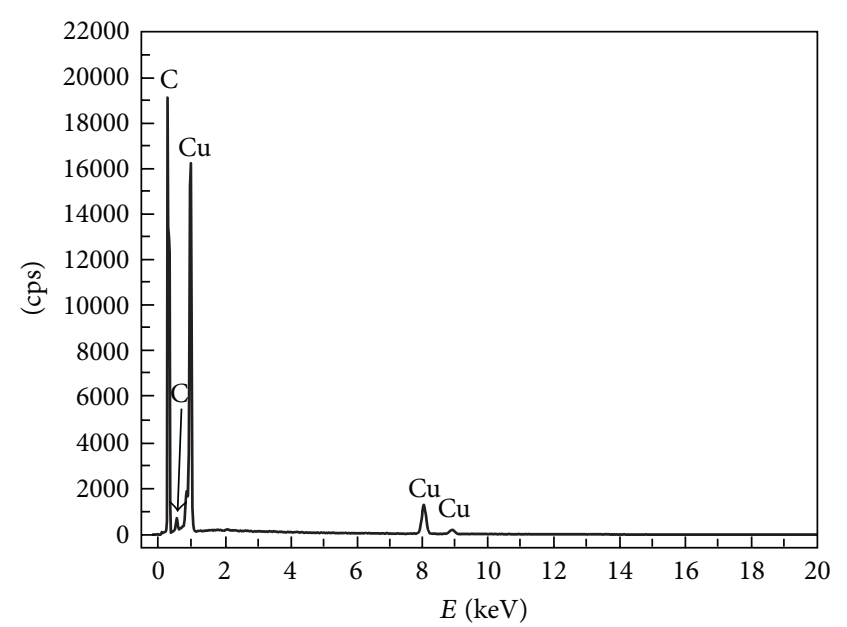

(a)

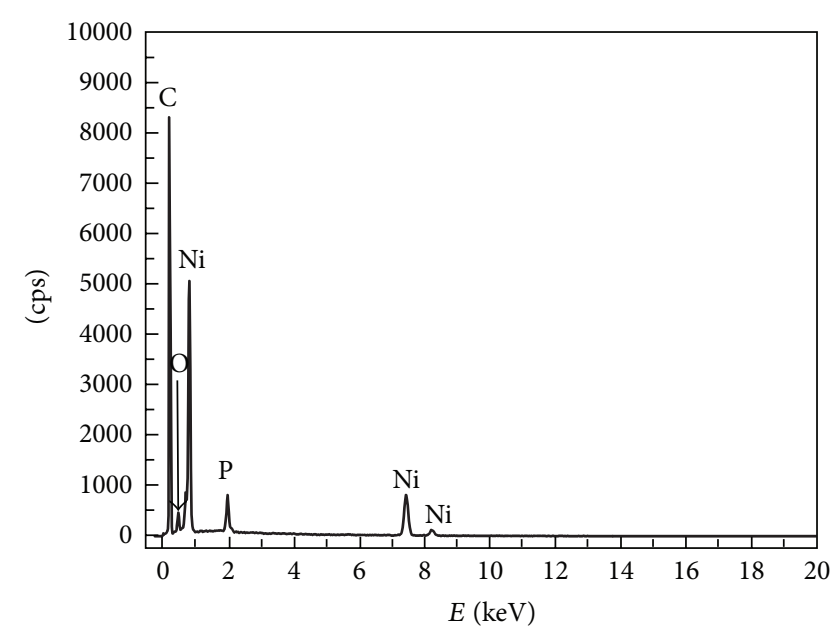

(b)

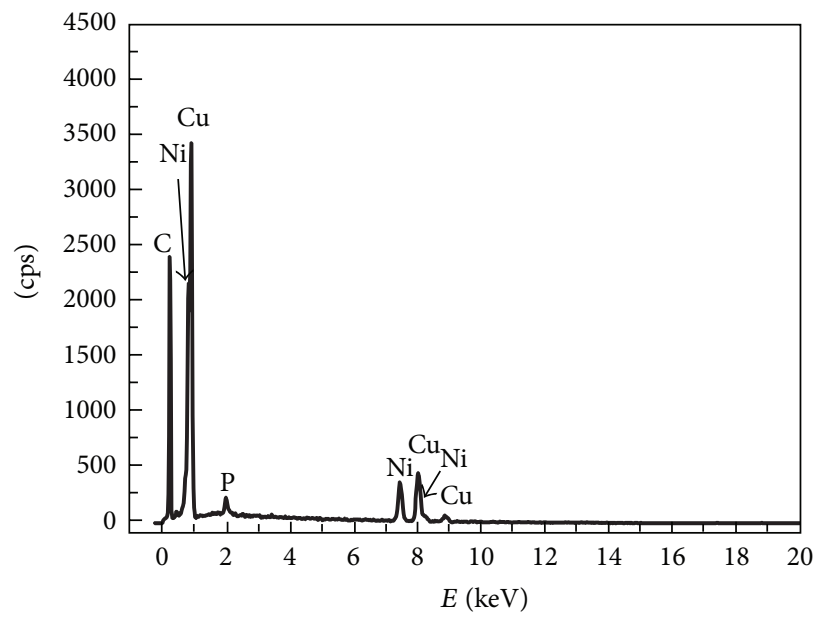

(c)

FIgUre 3: EDX spectra of (a) Cu deposited SWCNTs, (b) Ni deposited SWCNTs, and (c) Ni-Cu deposited SWCNTs.

TABLE 3: Element contents of EDX spectra for deposited SWCNTs.

\begin{tabular}{|c|c|c|c|c|c|c|}
\hline \multirow{2}{*}{$\begin{array}{l}\text { Sample } \\
\text { Element }\end{array}$} & \multicolumn{2}{|c|}{$\mathrm{Cu}$ deposited SWCNTs } & \multicolumn{2}{|c|}{ Ni deposited SWCNTs } & \multicolumn{2}{|c|}{ Ni-Cu deposited SWCNTs } \\
\hline & wt.\% & at.\% & wt.\% & at. $\%$ & wt.\% & at. $\%$ \\
\hline $\mathrm{C}$ & 52.52 & 80.86 & 55.49 & 81.02 & 31.07 & 67.45 \\
\hline $\mathrm{O}$ & 6.16 & 7.12 & 6.17 & 6.76 & 3.70 & 4.09 \\
\hline $\mathrm{Cu}$ & 41.32 & 12.02 & 0 & 0 & 40.52 & 17.14 \\
\hline $\mathrm{Ni}$ & 0 & 0 & 35.46 & 10.59 & 23.75 & 10.47 \\
\hline $\mathrm{P}$ & 0 & 0 & 2.87 & 1.63 & 0.96 & 0.84 \\
\hline
\end{tabular}

direction of priority crystal plane in $\mathrm{Cu}$-coating is $\left(\begin{array}{lll}2 & 2 & 0\end{array}\right)$, followed by $\left(\begin{array}{lll}2 & 0 & 0\end{array}\right)$ direction. It may be inferred that copper deposit grew outside on the SWCNTs.

In the diffraction pattern of Ni deposited SWCNTs corresponding to Figure 4(c), broad peaks are found at around 44.37, 51.92, and 76.73, corresponding to the ( $\left.\begin{array}{lll}1 & 1 & 1\end{array}\right)$, (2 00 ( 0 ), and (2 2 (2) planes of the fcc phase of nickel, respectively. The broad diffraction peak indicates a fine-grain nature of the $\mathrm{Ni}$-coating.
In the diffraction pattern of Ni-Cu deposited SWCNTs corresponding to Figure 4(d), five peaks are observed at approximately 41.6, 43.2, 52.3, 74.7, and 90, respectively. The peak at 43.2 was assigned to the (lll 111 ) plane of nickel, and the remaining four peaks at 41.6, 52.3, 74.7, and 90 correspond to the $\left(\begin{array}{lll}1 & 1 & 1\end{array}\right),\left(\begin{array}{lll}2 & 0 & 0\end{array}\right),\left(\begin{array}{lll}2 & 2 & 0\end{array}\right)$, and (3 11 1) planes of the fcc phase of copper, respectively.

By comparing the diffraction peaks of deposited SWCNTs with those of purified SWCNTs in Figure 4, it is confirmed 


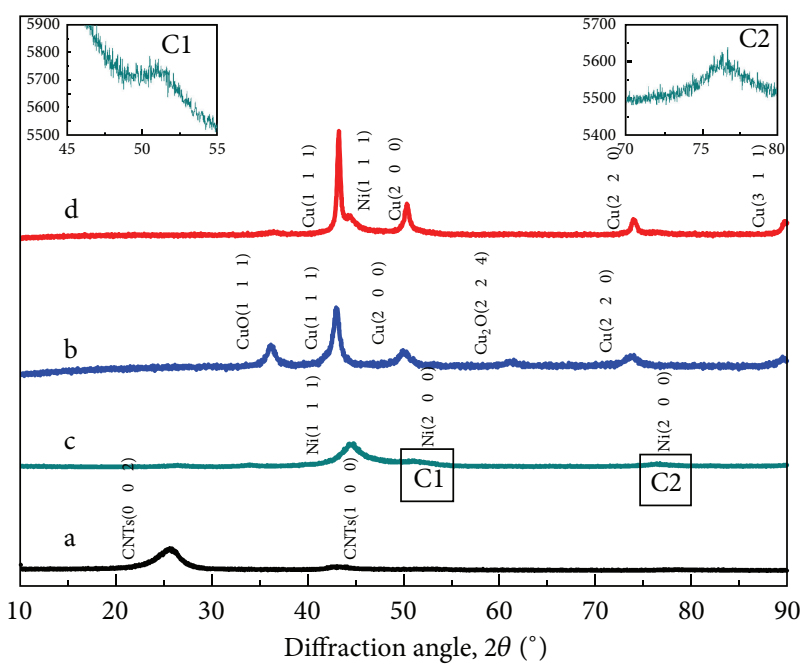

FIGURE 4: XRD patterns of (a) purified SWCNTs and (b) $\mathrm{Cu}$ deposited SWCNTs and (c) Ni deposited SWCNTs and (d) Ni-Cu deposited SWCNTs.

that layers of crystalline copper or nickel are newly introduced on surfaces of SWCNTs through electroless deposition. Furthermore, on comparison of the XRD results between Figures 4(b) and 4(d), no peak corresponds to $\mathrm{CuO}$ or $\mathrm{Cu}_{2} \mathrm{O}$ in Figure $4(\mathrm{~d})$, indicating that the Cu-coating on the $\mathrm{Ni}$ deposited SWCNTs is oxidized hardly, while the XRD results also clearly manifest that those of copper deposited SWCNTs are oxidized seriously and are consistent with the above EDX results.

In addition, it was found that the peak corresponding to the $\left(\begin{array}{lll}1 & 1 & 1\end{array}\right)$ plane improved and those corresponding to the $\left(\begin{array}{lll}2 & 0 & 0\end{array}\right)$ and $\left(\begin{array}{lll}2 & 2 & 0\end{array}\right)$ planes weakened clearly in Figure 4(d). It shows that the grain arrangements in $\mathrm{Cu}$ coating on the Ni deposited SWCNTs are more compact than those of copper deposited SWCNTs, because the former $\mathrm{Cu}$-coating is composed of $\left(\begin{array}{lll}3 & 1 & 1\end{array}\right)$ and the predominant $\left(\begin{array}{lll}1 & 1 & 1\end{array}\right)$ fcc planes, whose atomic arrangements are more intensive than those in the latter $\mathrm{Cu}$-coating. There exist obvious preferential oriented crystal textures in the $\mathrm{Cu}$ coating over Ni substrate, namely, epitaxy growth.

3.4. TG-DTA. Figure 5 shows the TGA and DTA curves of purified, copper deposited SWCNTs, Ni deposited SWCNTs, and $\mathrm{Ni}-\mathrm{Cu}$ deposited SWCNTs at temperature from 20 to $900^{\circ} \mathrm{C}$. The final temperature $\left(900^{\circ} \mathrm{C}\right)$ is sufficient for complete burning of SWCNTs, leaving metal oxide on the sample holder. Therefore the residual weights (\%) after the heating process show the metal oxide contents. The TGA curves in Figure 5(a) show that the ash content $(\mathrm{CuO})$ increased from $1.7 \mathrm{wt} \%$ (purified sample) to $47.2 \mathrm{wt} \%$ (copper deposited SWCNTs sample), whereas the ash content $(\mathrm{CuO}$ and $\mathrm{NiO}$ ) is $67.1 \mathrm{wt} \%$ (Ni-Cu deposited SWCNTs sample), and the ash content $(\mathrm{NiO})$ is $40.5 \mathrm{wt} \%$ ( $\mathrm{Ni}$ deposited SWCNTs sample). And the corresponding metal contents (weight\%) converted from metal oxide of copper deposited SWCNTs sample, Ni deposited SWCNTs sample, and Ni-Cu deposited SWCNTs sample are $41.7 \mathrm{wt} \%$ (copper), $34.8 \mathrm{wt} \%$ (nickel), and $57.3 \mathrm{wt} \%$ (nickel and copper), respectively.

The TGA curve of copper deposited SWCNTs (b) in Figure 5(a) shows a slight increase in weight at low temperatures. It coincides with two exothermic peaks of DTA curve (a) in Figure 5(b) at approximately $150^{\circ} \mathrm{C}$ and $290^{\circ} \mathrm{C}$, respectively. The first exothermic peak corresponds to the oxide of metallic copper into $\mathrm{Cu}_{2} \mathrm{O}$, and the second peak corresponds to the oxidization of $\mathrm{Cu}_{2} \mathrm{O}$ into $\mathrm{CuO}$, simultaneously to the oxidization of metallic copper into $\mathrm{Cu}_{2} \mathrm{O}$.

While the temperature corresponding to weight increment shows a significant rise for the TGA curve of $\mathrm{Ni}$ $\mathrm{Cu}$ deposited SWCNTs (d) in Figure 5(a), it also coincides with three exothermic peaks of DTA curve (d) in Figure 5(b) at higher temperature of approximately $230^{\circ} \mathrm{C}$, $370^{\circ} \mathrm{C}$, and $510^{\circ} \mathrm{C}$, respectively. The third exothermic peak can be explained by oxidation of nickel. It indicates the nickel improves the oxidation resistance of $\mathrm{Cu}-\mathrm{Ni}$ bilayers of coating.

The DTA curves (a) in Figure 5(b) corresponding to purified SWCNTs show exothermic peaks above $600^{\circ} \mathrm{C}$ are due to the SWCNTs oxidization, because it is accompanied by a sharp weight decrease in Figure 5(a). However, the degradation temperature of $\mathrm{Ni}-\mathrm{Cu}$ deposited SWCNTs sample was greatly reduced to $550^{\circ} \mathrm{C}$ (see DTA curve (a) in Figure 5(b)), and that of copper deposited SWCNTs sample was $570^{\circ} \mathrm{C}$ (see DTA curve (b) in Figure 5(b)), showing that the thermal stability of $\mathrm{Cu} / \mathrm{SWCNTs}$ samples declined. This is due to the catalytic effect of copper on the oxidization of SWCNTs. And the degradation temperature of electroless $\mathrm{Ni}$ deposited SWCNTs sample was increased to $690^{\circ} \mathrm{C}$, providing protection.

3.5. Mechanism on the Oxidation Resistance of $\mathrm{Ni}$-Cu Bimetallic Coating. Glyoxalic acid has an electric potential gap of more than $1.0 \mathrm{~V}$ under the conditions of between $\mathrm{pH} 0$ and $\mathrm{pH} 14$ during copper electroless plating [31]. The copper is inclined to be reduced under alkaline environment based on the thermodynamics principle. Besides the effective reaction of bivalent ion copper being reduced to metal copper by glyoxalic acid, there are many secondary reactions which produce $\mathrm{Cu}(\mathrm{OH})_{2}$ or $\mathrm{Cu}_{2} \mathrm{O}$ precipitation. The main secondary reactions are presented as follows:

Cannizzaro reaction:

$2 \mathrm{HCHO}+\mathrm{OH}^{-} \longrightarrow \mathrm{CH}_{3} \mathrm{OH}+\mathrm{HCOO}^{-}$

incomplete reduction:

$$
2 \mathrm{Cu}^{2+}+\mathrm{HCHO}+5 \mathrm{OH}^{-} \longrightarrow \mathrm{Cu}_{2} \mathrm{O} \downarrow+\mathrm{HCOO}^{-}+3 \mathrm{H}_{2} \mathrm{O}
$$

Cuprous oxide particles suspended in the plating bath will initiate a series of decomposition reactions:

$$
\begin{gathered}
\mathrm{Cu}_{2} \mathrm{O}+\mathrm{H}_{2} \mathrm{O} \longrightarrow 2 \mathrm{Cu}^{+}+2 \mathrm{OH}^{-} \\
\mathrm{Cu}_{2} \mathrm{O}+\mathrm{H}_{2} \mathrm{O} \longrightarrow \mathrm{Cu}^{0}+\mathrm{Cu}^{2+}+2 \mathrm{OH}^{-} \\
2 \mathrm{Cu}^{+} \longrightarrow \mathrm{Cu}^{0}+\mathrm{Cu}^{2+}
\end{gathered}
$$




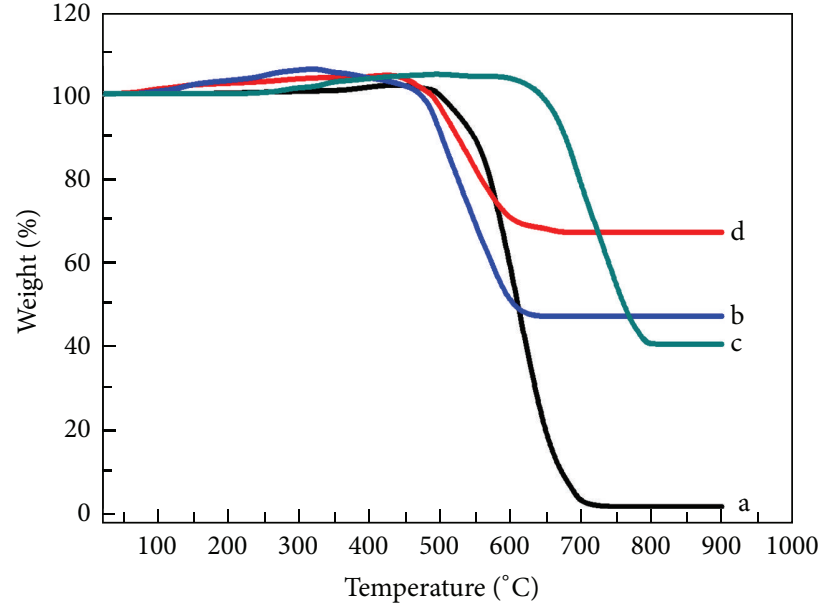

(a)

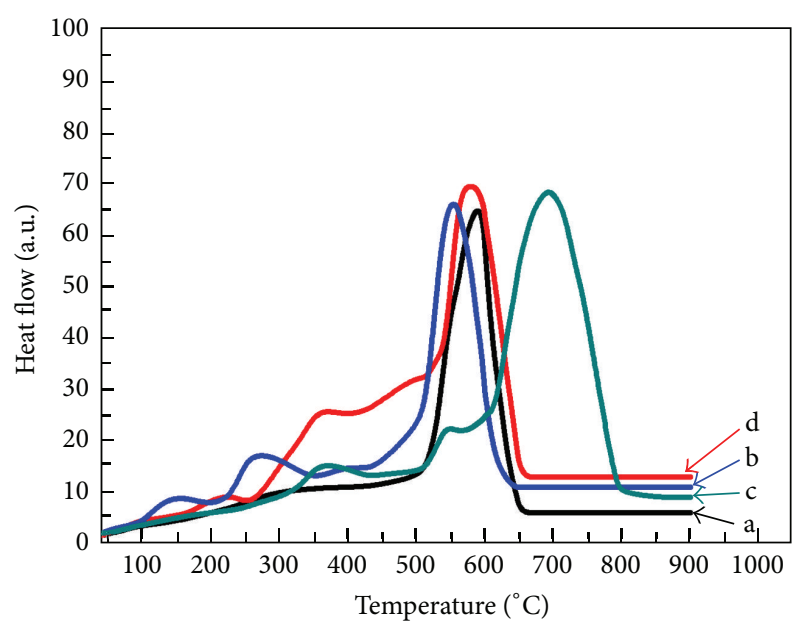

(b)

Figure 5: (a) TGA and (b) DTA curves of (a) purified SWCNTs and (b) Cu deposited SWCNTs and (c) Ni deposited SWCNTs and (d) Ni-Cu deposited SWCNTs.

The cuprous oxide and cupric hydroxide precipitation generated in the secondary reactions makes copper monolayers loose and coarse with a large amount of impurity of oxygen that exists in the forms of copper oxides $\left(\mathrm{CuO}\right.$ and $\left.\mathrm{Cu}_{2} \mathrm{O}\right)$.

The galvanic cell system formed while SWCNTs deposited with $\mathrm{Ni}$ were immersed into copper electroless plating bath and copper ions contacted with Ni-coating. Electrode potential of nickel is $-0.23 \mathrm{~V}$ versus SHE, while that of copper is $0.3402 \mathrm{~V}$ versus SHE. Copper ions were prevented from losing electrons, then avoided being copper oxides, and reduced to metal copper. That is,

$$
\begin{gathered}
\text { anode: } \mathrm{Ni} \longrightarrow \mathrm{Ni}^{2+}+2 \mathrm{e}^{-} \\
\text {cathode: } \mathrm{Cu}^{2+}+2 \mathrm{e}^{-} \longrightarrow \mathrm{Cu} \\
\mathrm{Cu}^{+}+\mathrm{e}^{-} \longrightarrow \mathrm{Cu}
\end{gathered}
$$

There were quite a few vacancies among the coarse grains of copper monolayers, and thus oxygen in the air had more access to copper coating through adsorption. $\mathrm{Cu}$-coating is prone to oxidation. Ni-Cu bilayers had fine and compact grains with few of the vacancies and defects, which prevented oxygen from permeating the coating.

\section{Conclusions}

In this study, the electroless coating technics was applied to prepare nickel-copper bilayers coated on SWCNTs. Original SWCNTs synthesized through CVD have been pretreated through the refluxing purification, sensitization, and activation process before electroless plating. For comparison, an electroless coating process depositing SWCNTs with monolayers of copper was also carried out. The results of SEM, EDS, XRD, and TG-DTA clearly confirmed that Ni-Cu bilayers had the fine and compact grains with higher purity and fewer of the vacancies than copper monolayers.
The method of electroless coating in this research could be applied to improve the wettability of the surface of SWCNTs reinforcement and increase the bonding strength of interfaces between SWCNTs and copper matrix of composite. Because of the omission of technic step of reducing copper oxide, this method has the characteristics of easy operation and control.

\section{Conflict of Interests}

The authors declare that there is no conflict of interests regarding the publication of this paper.

\section{Acknowledgments}

The authors are thankful for the financial support from the National Natural Science Foundation of China (Grant no. 5120514) and Hubei Provincial Key Laboratory of Green Materials for Light Industry (Grant no. 20132-15).

\section{References}

[1] S. Wang, R. Liang, B. Wang, and C. Zhang, "Load-transfer in functionalized carbon nanotubes/polymer composites," Chemical Physics Letters, vol. 457, no. 4-6, pp. 371-375, 2008.

[2] M. Estili and A. Kawasaki, "An approach to mass-producing individually alumina-decorated multi-walled carbon nanotubes with optimized and controlled compositions," Scripta Materialia, vol. 58, no. 10, pp. 906-909, 2008.

[3] W. M. Daoush, B. K. Lim, C. B. Mo, D. H. Nam, and S. H. Hong, "Electrical and mechanical properties of carbon nanotube reinforced copper nanocomposites fabricated by electroless deposition process," Materials Science and Engineering A, vol. 513-514, pp. 247-253, 2009.

[4] H. Li, A. Misra, Y. Zhu, Z. Horita, C. C. Koch, and T. G. Holesinger, "Processing and characterization of nanostructured $\mathrm{Cu}$-carbon nanotube composites," Materials Science and Engineering $A$, vol. 523, no. 1-2, pp. 60-64, 2009. 
[5] P. V. Trinh, T. B. Trung, N. B. Thang et al., "Calculation of the friction coefficient of $\mathrm{Cu}$ matrix composite reinforced by carbon nanotubes," Computational Materials Science, vol. 49, no. 4, pp. S239-S241, 2010.

[6] Y. L. Yang, Y. D. Wang, Y. Ren et al., "Single-walled carbon nanotube-reinforced copper composite coatings prepared by electrodeposition under ultrasonic field," Materials Letters, vol. 62, no. 1, pp. 47-50, 2008.

[7] S. Cho, K. Kikuchi, and A. Kawasaki, "On the role of amorphous intergranular and interfacial layers in the thermal conductivity of a multi-walled carbon nanotube-copper matrix composite," Acta Materialia, vol. 60, no. 2, pp. 726-736, 2012.

[8] Y. Peng and Q. Chen, "Ultrasonic-assisted fabrication of highly dispersed copper/multi-walled carbon nanotube nanowires," Colloids and Surfaces A: Physicochemical and Engineering Aspects, vol. 342, no. 1-3, pp. 132-135, 2009.

[9] E. Khaleghi, M. Torikachvili, M. A. Meyers, and E. A. Olevsky, "Magnetic enhancement of thermal conductivity in coppercarbon nanotube composites produced by electroless plating, freeze drying, and spark plasma sintering," Materials Letters, vol. 79, pp. 256-258, 2012.

[10] B. Wang, J. Hao, Q. Li, and H. Li, "New insights into thermal conduction mechanisms of multi-walled carbon nanotube/ionic liquid suspensions," International Journal of Thermal Sciences, vol. 83, no. 1, pp. 89-95, 2014.

[11] S. Berber, Y.-K. Kwon, and D. Tománek, "Unusually high thermal conductivity of carbon nanotubes," Physical Review Letters, vol. 84, no. 20, pp. 4613-4616, 2000.

[12] P. Kim, L. Shi, A. Majumdar, and P. L. McEuen, "Thermal transport measurements of individual multiwalled nanotubes," Physical Review Letters, vol. 87, no. 21, Article ID 215502, 2001.

[13] J. Lin, J. Shen, T. Wang et al., "Enhancement of photocatalytic properties of $\mathrm{TiO}_{2}$ nanobelts through surface-coarsening and surface nanoheterostructure construction," Materials Science and Engineering B, vol. 176, no. 12, pp. 921-925, 2011.

[14] K. A. Nazari and S. G. Shabestari, "Effect of micro alloying elements on the interfacial reactions between molten aluminum alloy and tool steel," Journal of Alloys and Compounds, vol. 478, no. 1-2, pp. 523-530, 2009.

[15] X. Luo, S. Luo, Y. Zhan, H. Shu, Y. Huang, and X. Tu, "Novel $\mathrm{Cu}$ (II) magnetic ion imprinted materials prepared by surface imprinted technique combined with a sol-gel process," Journal of Hazardous Materials, vol. 192, no. 3, pp. 949-955, 2011.

[16] A. Jitianu, T. Cacciaguerra, R. Benoit, S. Delpeux, F. Béguin, and S. Bonnamy, "Synthesis and characterization of carbon nanotubes- $\mathrm{TiO}_{2}$ nanocomposites," Carbon, vol. 42, no. 5-6, pp. 1147-1151, 2004.

[17] V. M. Krishna, A. Abilarasu, T. Somanathan, and N. Gokulakrishnan, "Effective synthesis of well graphitized high yield bamboo-like multi-walled carbon nanotubes on copper loaded $\alpha$-alumina nanoparticles," Diamond and Related Materials, vol. 50, pp. 20-25, 2014.

[18] G. M. Ilari, N. Kränzlin, R. Longtin et al., "Single-step functionalization of vertically aligned MWCNTs with $\mathrm{Cu}$ and $\mathrm{Ni}$ by chemical reduction of copper and nickel acetyl acetonate in benzyl alcohol," Carbon, vol. 73, pp. 146-154, 2014.

[19] L. Qingming, N. Kazuaki, K. Kensuke, and O. Masazumi, "Effects of reaction parameters on the preparation of submicron $\mathrm{Cu}$ particles by liquid phase reduction method and the study of reaction mechanism," Powder Technology, vol. 241, pp. 98-104, 2013.
[20] M. Kapkowski, P. Bartczak, M. Korzec et al., " $\mathrm{SiO}_{2^{-}}, \mathrm{Cu}-$, and $\mathrm{Ni}$-supported $\mathrm{Au}$ nanoparticles for selective glycerol oxidation in the liquid phase," Journal of Catalysis, vol. 319, pp. 110-118, 2014.

[21] M. Gopiraman, S. G. Babu, Z. Khatri et al., "An efficient, reusable copper-oxide/carbon-nanotube catalyst for N-arylation of imidazole," Carbon, vol. 62, pp. 135-148, 2013.

[22] M. Yin, C.-K. Wu, Y. Lou et al., "Copper oxide nanocrystals," Journal of the American Chemical Society, vol. 127, no. 26, pp. 9506-9511, 2005.

[23] J. Zhang, J. Liu, Q. Peng, X. Wang, and Y. Li, "Nearly monodisperse $\mathrm{Cu} 2 \mathrm{O}$ and $\mathrm{CuO}$ nanospheres: preparation and applications for sensitive gas sensors," Chemistry of Materials, vol. 18, no. 4, pp. 867-871, 2006.

[24] X. Zhang, H. Ying, and L. Geng, "The preparation method of carbon nanotubes-copper oxide composite powder," China: 200910311249,2010-06-02, 2010.

[25] L. Xu, W. Pan, W. Li et al., "Thermal expansion of MWCNTreinforced copper composite," Transactions of Nonferrous Metals Society of China, vol. 1, 2007.

[26] C. Z. Jinmei Wang, "Ni-Cu plated CNTs and the properties of anti-electromagnetic wave," Acta Materiae Compositae Sinica, vol. 22 , no. 6, pp. 54-58, 2006.

[27] K. Kiani, "Nonlocal continuous models for forced vibration analysis of two- and three-dimensional ensembles of singlewalled carbon nanotubes," Physica E: Low-Dimensional Systems and Nanostructures, vol. 60, pp. 229-245, 2014.

[28] M. F. Islam, E. Rojas, D. M. Bergey, A. T. Johnson, and A. G. Yodh, "High weight fraction surfactant solubilization of singlewall carbon nanotubes in water," Nano Letters, vol. 3, no. 2, pp. 269-273, 2003.

[29] R. Haggenmueller, S. S. Rahatekar, J. A. Fagan et al., “Comparison of the quality of aqueous dispersions of single wall carbon nanotubes using surfactants and biomolecules," Langmuir, vol. 24, no. 9, pp. 5070-5078, 2008.

[30] X. Yang, F. Qiao, X. Zhu, P. Zhang, D. Chen, and A. C. To, "Coalescence of parallel finite length single-walled carbon nanotubes by heat treatment," Journal of Physics and Chemistry of Solids, vol. 74, no. 3, pp. 436-440, 2013.

[31] X. Jiang and W. Shen, The Fundamental and Practice of Electroless Plating, National Defence Industry Press, 2000. 

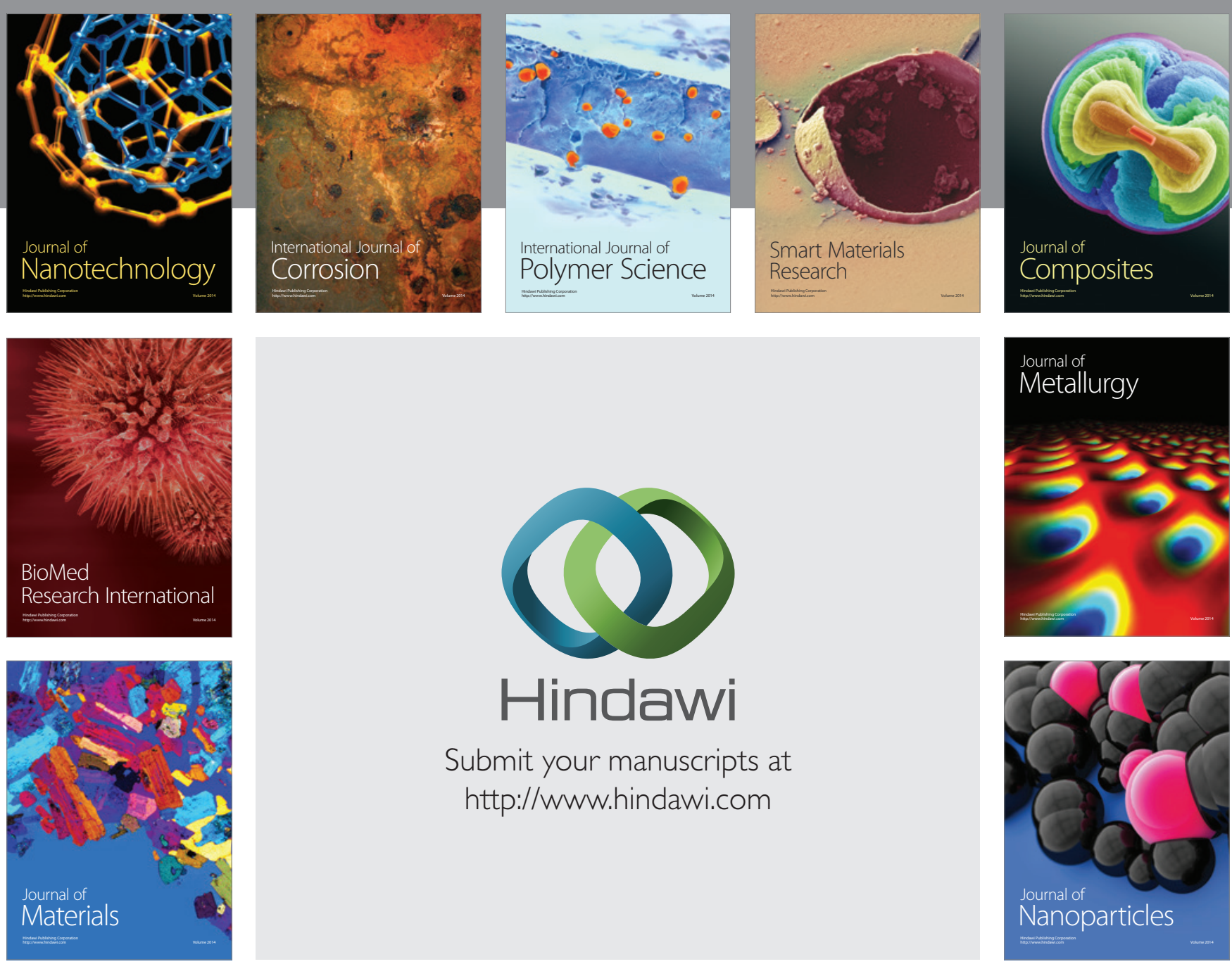

Submit your manuscripts at http://www.hindawi.com
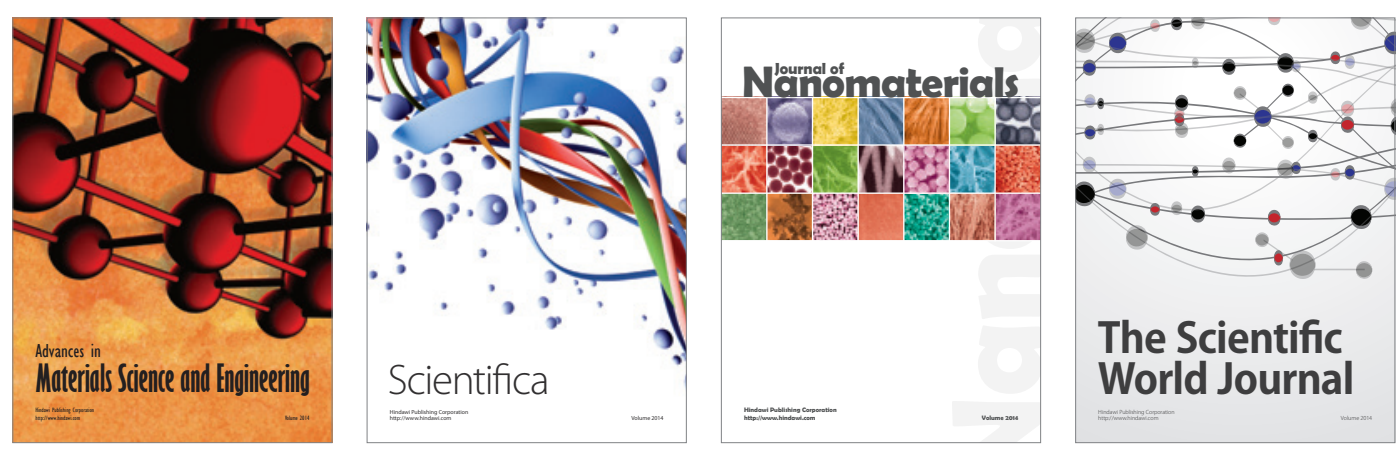

\section{The Scientific World Journal}
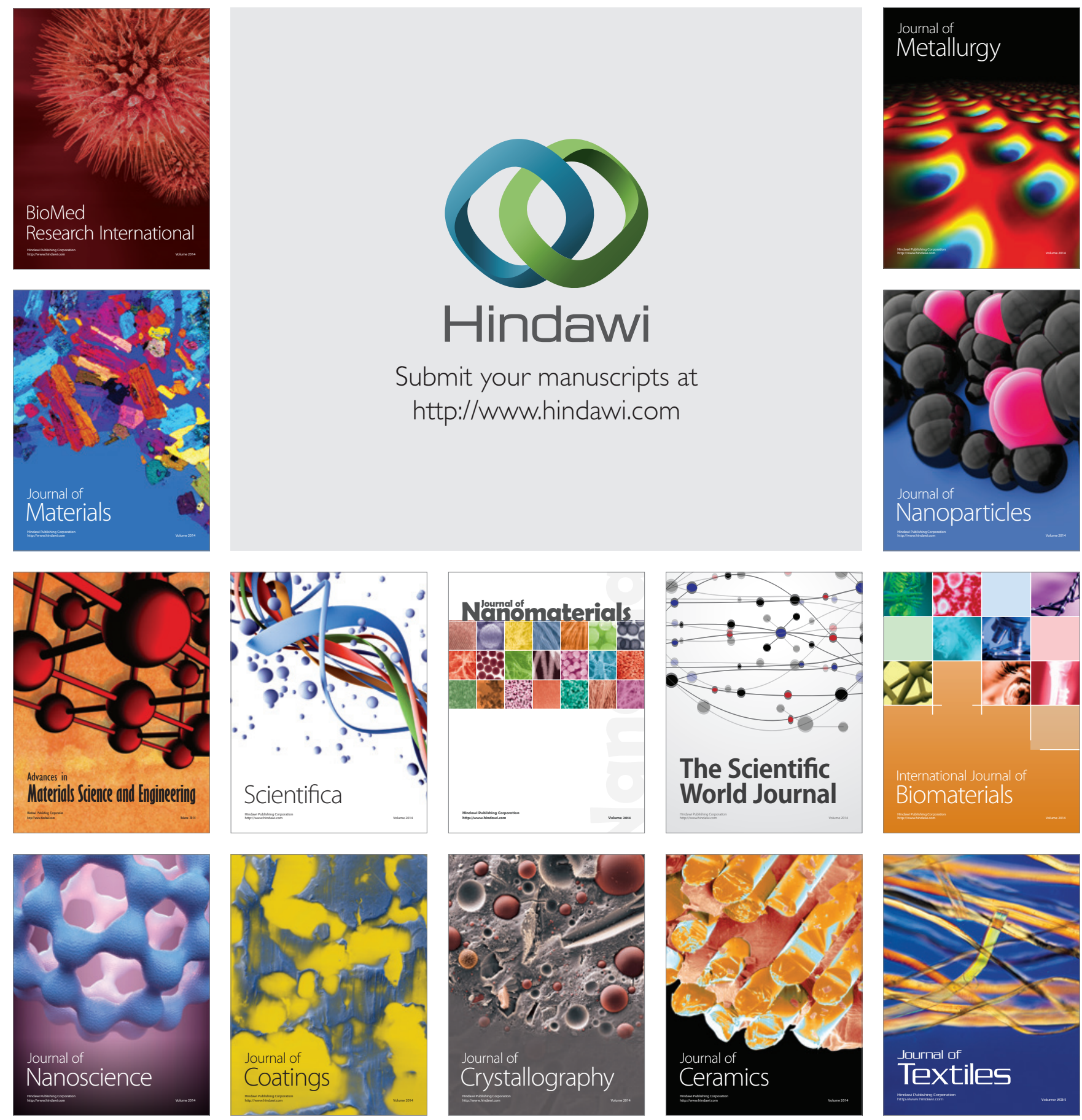